\title{
A influência da neotectônica nos sistemas costeiro, fluvial e lacustre na região costeira oriental norte do $\mathrm{RN}$-Brasil
}

\author{
The influence of neotectonic in coastal, river and lake systems in the eastern coastal region \\ northern RN- Brazil \\ Camara $^{1}$, M. R.; Cestaro ${ }^{1}$, L. A.; Louzeiro $^{2}$, A. S. \\ Rodcam1987@gmail.com;
}

\begin{abstract}
Resumo
O presente trabalho foi uma pesquisa realizada na região costeira oriental norte do Rio Grande do Norte, abordando os municípios de Touros, Pureza, Rio do Fogo, Maxaranguape e Ceará Mirim. Cujo o objetivo foi identificar o grau de influência da forçante endógena da neotectônica nas unidades geoambientais costeiras, fluviais e lacustres, através de vistoria a campo, análise de imagens de satélite e de radar, além de geoprocessamento e análises multitemporais com softwares de ArcGIS 10.3 e ERMapper 7.1. Tal estudo identificou o forte controle tectônico nos ambientes fluviais e lacustres, que possuem uma orientação paralela às principais falhas identificadas na região costeira oriental sul do Estado. No sistema costeiro, também foram identificados a influência dessa forçante em corpos dunares que apresentaram sua forma perpendicular à direção dos ventos alísios que controlam os campos de dunas. Enquanto que na faixa de praia, foi identificado uma forte variação de sua extensão devido ao afloramento de falésias que são o resultado do soerguimento das rochas areníticas da Formação Barreiras, que por sua vez é uma grande evidencia de um sistema de horst-graben nessa área de estudo.

Palavras-chave: Neotectônica, geomorfologia costeira, geoprocessamento.
\end{abstract}

\begin{abstract}
This study was a survey in the eastern coastal region north of Rio Grande do Norte, covering the municipalities of Bulls, Purity, River of Fire, Maxaranguape and Ceará Mirim. Whose goal was to identify the degree of influence of endogenous forcing the neotectonics in coastal geo-environmental units, river and lake, through survey the field, satellite imagery analysis and radar, as well as geoprocessing and multi-temporal analysis with ArcGIS 10.3 software and ERMAPPER 7.1. This study identified the strong tectonic control in fluvial and lacustrine environments, which have a parallel orientation to the main deficiencies identified in the eastern coastal region south of the state. In the coastal system were also identified the influence of this forcing in dune bodies presented their perpendicular to the direction of the trade winds that control dune fields. While on the beach strip, a strong variation of its length due to the outcrop of cliffs that are the result of the uplift of sandstone rocks of Barreiras was identified, which in turn is a great evidence of a horst-graben system in this area of study.
\end{abstract}

Keywords: Neotectonics, coastal geomorphology, geoprocessing.

\section{INTRODUÇÃO}

O termo "neotectônica" foi empregado, pela primeira vez, em 1948 por V. A. Obruchev para definir os movimentos da crosta terrestre que se instalaram durante os períodos do Terciário Superior (Mioceno e Plioceo) e do Quaternário, e que assumiram um papel decisivo na formação da topografia contemporânea. E. V. Jain (1973), afirmou que o advento dos estudos neotectônicos representa um importante fator de avanço para as pesquisas em geotectônica, pois permite a aplicação do princípio do atualismo, através da observação real das feições tectônicas que eram até então, em grande parte, inferidas.

Já Trifonov (1989), citou três circunstâncias que demonstram a importância da neotectônica para as ciências geográficas e geológicas: A possibilidade de observar, medir e datar de maneira

\footnotetext{
${ }^{1}$ Michel Rodrigues Camara, Pós Graduação de Engenharia Civil, Universidade Federal do Rio Grande do Norte, Natal-RN, Brasil

${ }^{1}$ Luiz Antonio Cestaro, Pós Graduação em Geografia, Universidade Federal do Rio Grande do Norte, Natal-RN, Brasil

${ }^{2}$ Andreza dos Santos Louzeiro, Pós Graduação em Geografia, Universidade Federal do Rio Grande do Norte, Natal-RN, Brasil
} 
mais precisa os movimentos tectônicos cenozóicos; a sismicidade e os campos geofísicos, estabelecendo a relação da tectônica superficial com a estrutura profunda e suas transformações, tornando-se possível então construir modelos tri-dimensionais; e por último, a comparação da tectônica ativa com as manifestações cenozoicas pretéritas, permite correlacionar as consequências dos eventos em escalas de tempo geológico média e real, bem como identificar a complexidade de seus desenvolvimentos.

Vale salientar também a sua importância econômica, uma vez que a reconstituição da geometria e atitude de feições deformacionais pode representar uma importante contribuição na quantificação para evolução neotectônica, geomorfológica e paisagística de uma região, tanto quanto fornecer parâmetros para caracterização de aquíferos e reservatórios petrolíferos, em subsuperfície.

O objetivo desse trabalho é identificar a influência da neotectônica nos sistemas costeiros, fluviais e lacustres, comparar tais influências com as das forçantes exógenas, caracterizadas pelos ventos, clima, deriva litorânea, entre outros e a partir daí, analisar o grau de importância desse elemento da forçante endógena para os sistemas dinâmicos em estudo na região costeira oriental norte do RN-Brasil.

\section{METODOLOGIA}

A pesquisa foi desenvolvida em toda a região norte do litoral oriental do Rio Grande do Norte, abrangendo os municípios de Touros, Pureza, Rio do Fogo, Maxaranguape e Ceará-Mirim. Esse estudo partiu dos conceitos geomorfológicos, neotectônicos e de fontes bibliográficas sobre a área de estudo para subsidiar a caracterização da influência tectônica sobre a paisagem dos ambientes em estudo. Além disso, utilizou-se de técnicas de geoprocessamento e pesquisa de campo para atingir o objetivo proposto.

A etapa de planejamento (Gabinete) também foi feita a partir da coleta de materiais cartográficos, imagens de satélite e arquivos vetoriais existentes da área de estudo. Com isso, foram utilizados os seguintes materiais: Cartas da Superintendência do Desenvolvimento do Nordeste SUDENE (2003) em formato CAD; Malha digital dos municípios do Rio Grande do Norte (2010) disponível no sítio do Instituto Brasileiro de Geografia e Estatística - IBGE; Imagem de satélite obtidas através do software Google Earth PRO do ano de 2014; Ortofotocartas (2006), escala 1:25000 do projeto PRODETUR; Arquivos vetoriais (Geomorfologia) em formato shapefile do IDEMA (2008); Imagem de satélite Shuttle Radar Topography Mission (SRTM), com resolução espacial de 30 metros, obtidas no site da NASA; Uso do sistema de posicionamento global (Global 
Positioning System, GPS-Navegação); Software ArcGIS versão trial 10.3.1, Er Mapper 7.1; Caderneta de campo para observações e Câmera fotográfica.

Após a obtenção dos materiais citados, os arquivos vetoriais foram espacializados e convertidos no software ArcGIS para o arquivo do tipo shapefile e reprojetados no sistema de coordenadas projetadas UTM, zona 25 M - DATUM SIRGAS2000. Já as imagens de satélite capturadas no Google Earth PRO foram georreferenciadas no ArcGIS utilizando como base as ortofotocartas reprojetadas na escala 1:25000 de 2006. O Modelo Digital de Terreno (MDT) foi gerado com base nas imagens de satélite SRTM e de Radar de resolução espacial de 30 metros, para um maior detalhe do comportamento da morfologia do relevo.

Desta forma, a partir dos arquivos vetoriais e imagens de satélite, foram elaborados os mapas de localização, altimétrico e geomorfológico considerados como "Mapa Base". Por meio dessa base cartográfica as unidades geoambientais foram delimitadas e a influência do controle tectônico nelas foi identificado e caracterizado através da interpretação visual sobre as imagens de satélite, ortofotocartas, curvas de níveis e MDT, para posterior verificação in loco para validação, reavaliação e correções das informações contidas no mapa.

Foi feita visita in loco, nos dias 23 de maio e 14 de junho de 2016. Com uso do GPS de navegação, os arquivos vetoriais referentes às vias de acesso, comunidades, corpos hídricos e as unidades geoambientais foram carregadas no equipamento, como também, pontos pré-selecionados para esclarecer dúvidas nos locais e validar as informações contidas nos mapas.

Após as verificações in loco, as informações coletadas contribuíram para que fossem realizadas as correções necessária nos mapas e a demarcação do controle tectônico sobre as unidades geoambientais na área em estudo.

\section{RESULTADOS E DISCUSSÃO}

Em estudos anteriores, Bezerra, Amaro e Vitta-Finzi (2001) observaram na zona costeira oriental do RN, a ocorrência de duas zonas de cisalhamentos, de trends a $\mathrm{NE}$ e NO, com deslizamento dextral e sinistral, relatando uma tectônica extensional e deformação transtracional respectivamente (Amaro 1998). O mergulho das foliações miloníticas são principalmente subverticais e as zonas miloníticas exibem evidencias de reativação frágil previsto pelas brechas de falha, veios de cataclasitos e de pseudotaquilitos, e a forma do embasamento onde a reativação cenozóica ocorreu.

Além disso, Bezerra, Amaro e Vitta-Finzi (2001) observaram que ao longo da planície costeira, muitas formas de falhas estão limitadas entre grabens e horsts que são responsáveis pela organização estrutural e morfológica costeira. 
Ao longo da planície costeira, pronunciadas falhas esclarecem a contemporaneidade com a deposição cenozóica. Foram reconhecidos 3 principais sets de falha com as direções: $040^{\circ}-060^{\circ}$ $\mathrm{NE}, 300^{\circ}-320^{\circ} \mathrm{NW}$ e $350^{\circ}-010^{\circ} \mathrm{N}$ (figura 1) novamente no Pleistoceno - Holoceno (Mabesoone e Lobo, 1980).

Porém tais estudos ficaram limitados desde o município de Ceará Mirim até o sul da região oriental costeira do Estado, ausentando a porção norte de estudos desse contexto, e a presente pesquisa comprova que a atividade neotectônica também ocorre na porção norte costeira do Estado.

Os estudos realizados nessa área identificaram que tanto o sistema costeiro, quanto o fluvial e o lacustre são fortemente controlados pela neotectônica, uma vez que os rios, lagoas, dunas e até uma porção praial se comporta de forma peculiar e diferente das demais unidades geoambientais que sofrem influência direta dos ventos, da deriva litorânea e do clima.

Ao analisar a área de estudo, foi notado nos principais sistemas fluviais dos cinco municípios costeiros da porção oriental norte costeira do RN - denominados de rio do Saco, Das Piranhas e Córrego Carro Quebrado no município de Touros; os rios das Curicacas, Tatu e córrego do Arrepiado em Pureza; os rios Punaú e D’água Colônia em Rio do Fogo; rio Maxaranguape e riacho Seco em Maxaranguape; e por últimos, o rio Maturaia de Cima e riachão em Ceará Mirím um forte controle neotectônico e ao avaliar a orientação deles, observou-se que os mesmos possuem curvas fortemente angulosas que estão paralelos ao trend das principais falhas encontradas na porção oriental do Estado do RN, de direção nordeste - sudoeste e sudeste - noroeste, inclusive com as mesmas atitudes identificadas em trabalhos anteriores para porção sul da costa oriental do $\mathrm{RN}$, de direções $040^{\circ}-060^{\circ} \mathrm{NE}$ e $300^{\circ}-320^{\circ} \mathrm{NW}$ (Figura 2). 


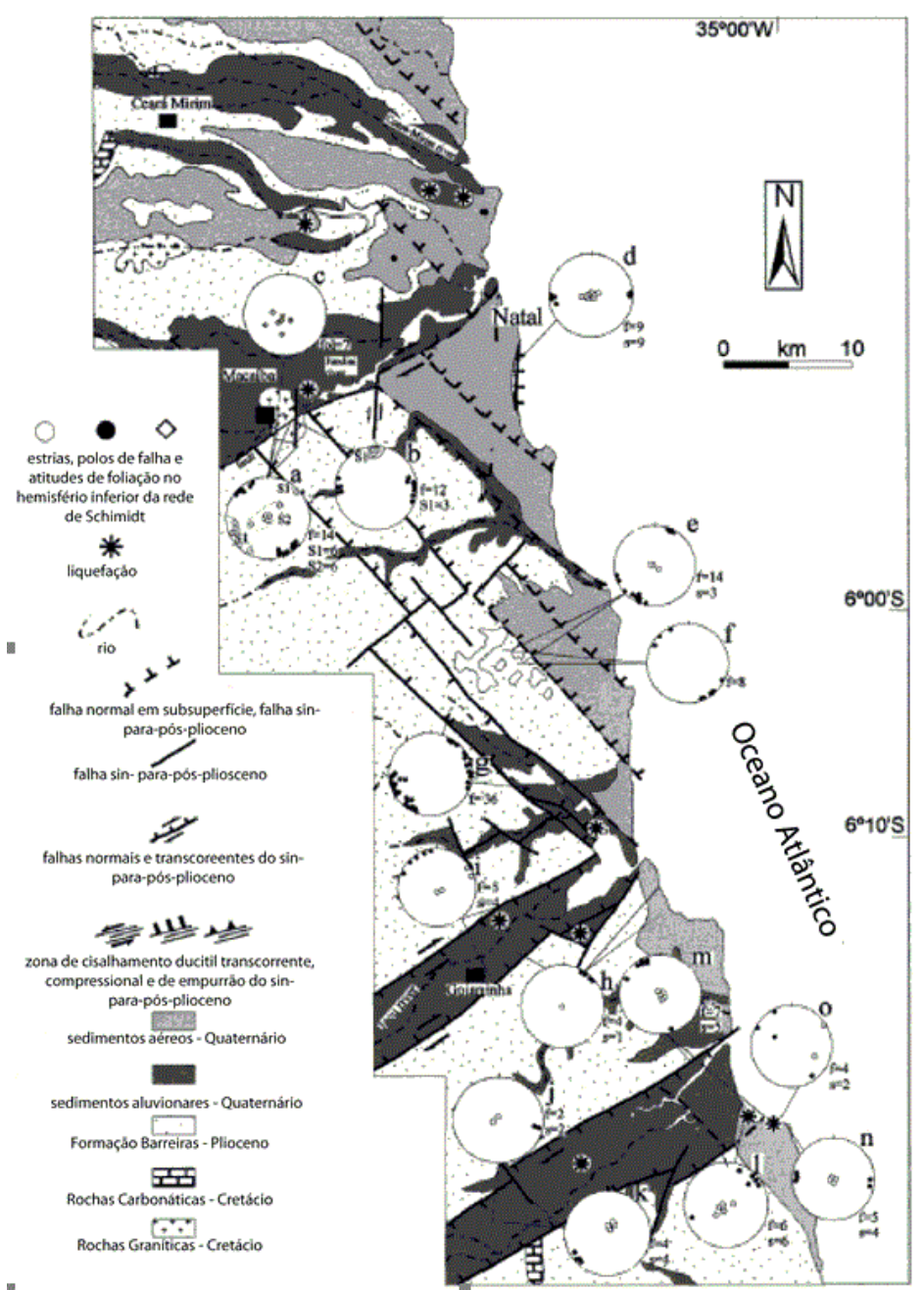

Figura 01. Detalhe da cobertura sedimentar mostrando os stereonets e a direção das principais falhas; S1, primeira geração de estrias; S2 segunda geração de estrias; S, estrias; f, polo da falha. A cidade de Natal foi omitida para melhor clareza. Fonte: Modificado de Bezerra, Amaro e Vita-Finzi, (2001).

Em comparação entre a influência da forçante endógena com relação as forçantes exógenas, o controle dessas últimas sobre os rios é muito menor, sendo limitado pelas dunas que estão paralelas a direção do vento e são movimentadas pelo mesmo em áreas muito pontuais. Uma das evidencias foi encontrada na desembocadura do rio Punaú que sofre total controle desses depósitos eólicos holocênicos dinâmicos que já deslocou a posição de sua desembocadura três vezes em menos de cinco anos, como observado na análise multitemporal através do software Er Mapper 7.1 realizada entre os anos de 2006, 2009 e 2011 (Figura 3). 


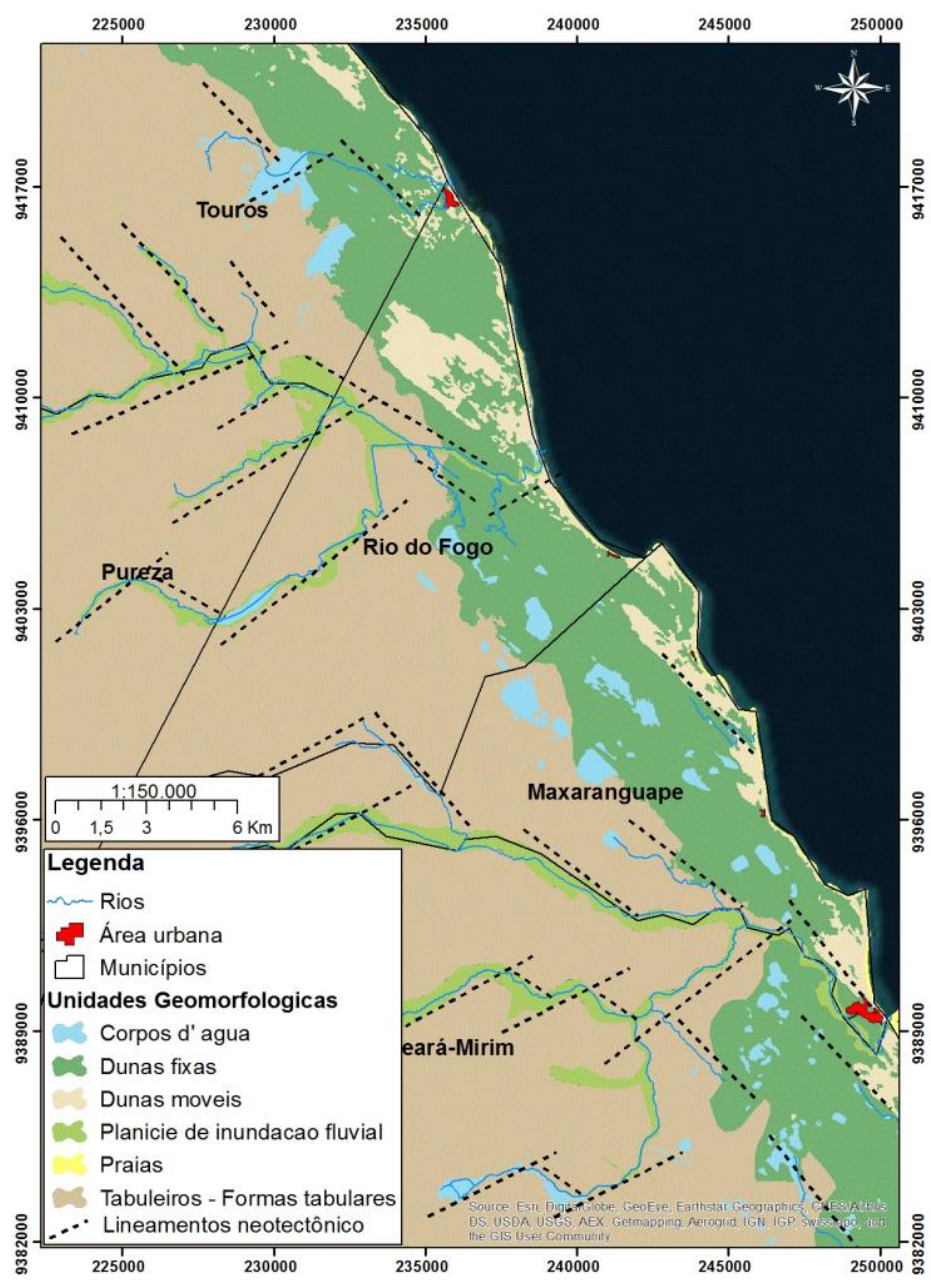

Figura 02. Lineamentos com attitude semelhante às das falhas e estruturas neotectônicas encontrados na porção sul da costa oriental do RN, com direções $040^{\circ}-060^{\circ} \mathrm{NE}$ e $300^{\circ}-320^{\circ} \mathrm{NW}$, controlando fortemente os principais canais fluviais dos municípios de Touros, Pureza, Rio do Fogo, Maxaranguape e Ceará Mirim. Fonte: Camara (2016)

Já na análise do sistema lacustre, também foi encontrado evidencias marcantes nas principais lagoas da área de estudo, como as lagoas do Boqueirão e do Coelho em Touros, lagoas do Mutuca e Cotias em Rio do Fogo e a lagoa Grande e da Cotia em Maxaranguape com semelhante morfologia onde as bordas lacustres na porção norte se encontram todas retilíneas e obedecendo a direção nordeste - sudoeste (Figuras 2 e 5), inclusive com cordões dunares de mesma orientação em uma região onde as dunas e lagoas são controladas principalmente pela força exógena dos ventos, com orientações sudeste - noroeste (Figura 5), além da lagoa do Católe que possui seu corpo alongado também obedecendo a orientação das principais falhas da região (Figura 2).

Em vistoria de campo e pelas imagens de satélite, ficou evidente que mesmo o vento transportando os sedimentos com direção noroeste-sudeste, as dunas vegetadas nas bordas das lagoas encontram-se perpendiculares, com orientação nordeste-sudoeste. Porém, a influência da 
neotectônica é mais suave, uma vez que os ventos possuem forte controle na morfodinâmica dunar e costeira (Figura 5).
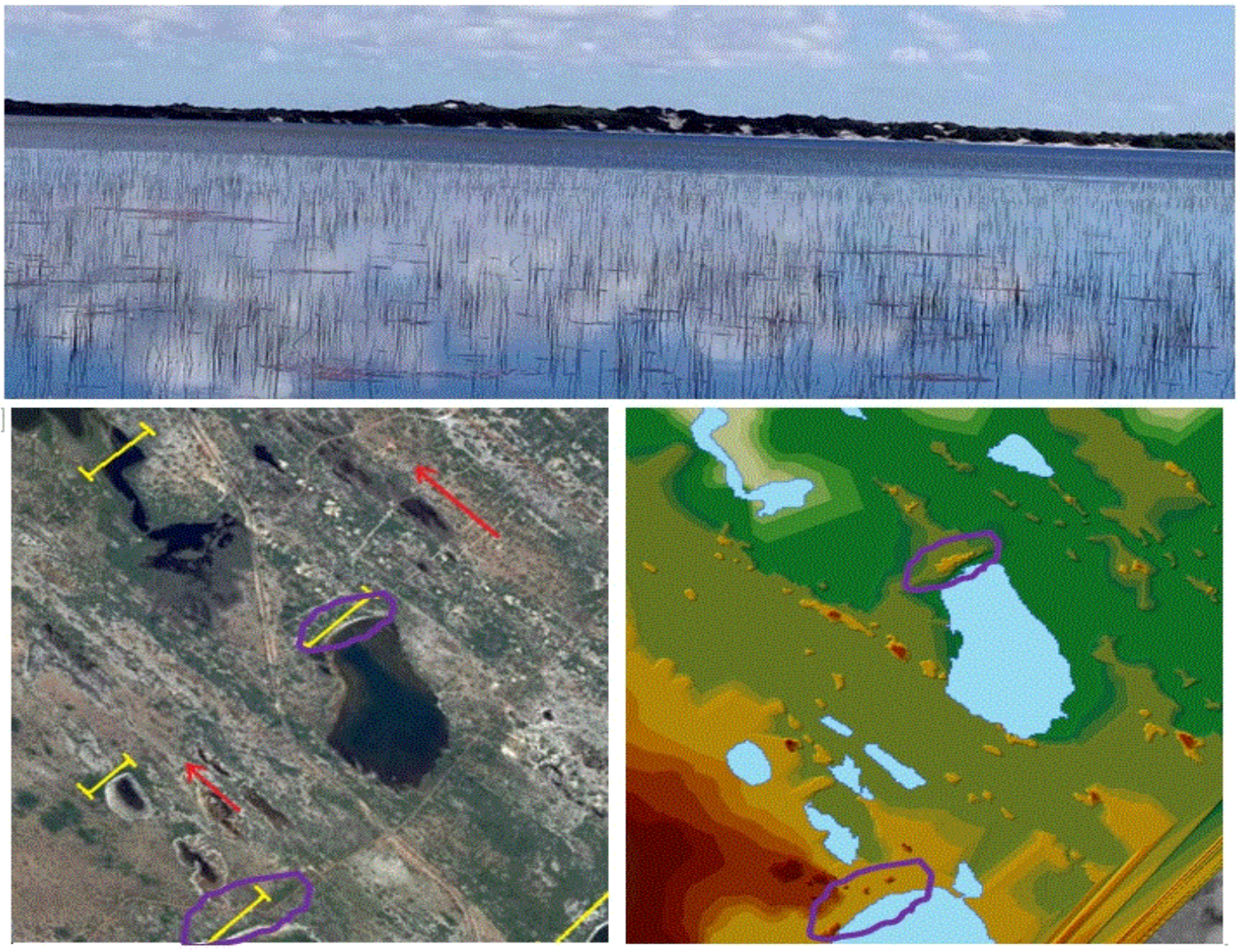

Figura 05. 05A mostra a duna vegetada paralela a borda da lagoa da Mutuca em Rio do Fogo, com orientação NESO, perpendicular ao do vento. 05B apresentando em imagem de satélite do Google Earth, a localização das dunas umas das dunas que estão sofreram controle tectônico, demarcadas em círculos violetas, com orientação apresentado em setas na cor amarelo, enquanto que a direção dos ventos alísios está apresentado em setas na cor vemelho. 05C mostra a morfologia desses corpos dunares em um modelo digital de elevação criado a partir de imagens de radar SRTM. Fonte: Camara (2016)

Fora as dunas, ainda no sistema costeiro, também foi observado uma forte variação da extensão da faixa de praia no município do Rio do Fogo, entre as porções sul, centro e norte. No sul a explicação para o estreitamento costeiro se dá por conta da própria morfologia em si, uma vez que entre os municípios de Rio do Fogo e Maxaranguape existe um pontal posicionado em um ângulo específico na diagonal com a deriva litorânea, favorecendo a formação de um vortex que intensifica o processo de erosão nesse local. Enquanto que nas porções do centro para norte ocorre uma diminuição da faixa praial de 155,80 metros na localidade ao sul do rio Punaú para 11 metros a $3 \mathrm{~km}$ ao norte do mesmo rio (Figura 6). Mesmo o rio depositando na praia sedimentos fluviais que são carreados para o norte do município pela deriva litorânea e pela corrente de maré, a faixa de praia continua bastante encurtada, e pela análise e interpretação do contexto local, uma das possíveis razões encontradas é devido a influência do afloramento de uma falésia viva que chega a alcançar 4 metros de altura, limitando essa faixa praial (Figura 7). 


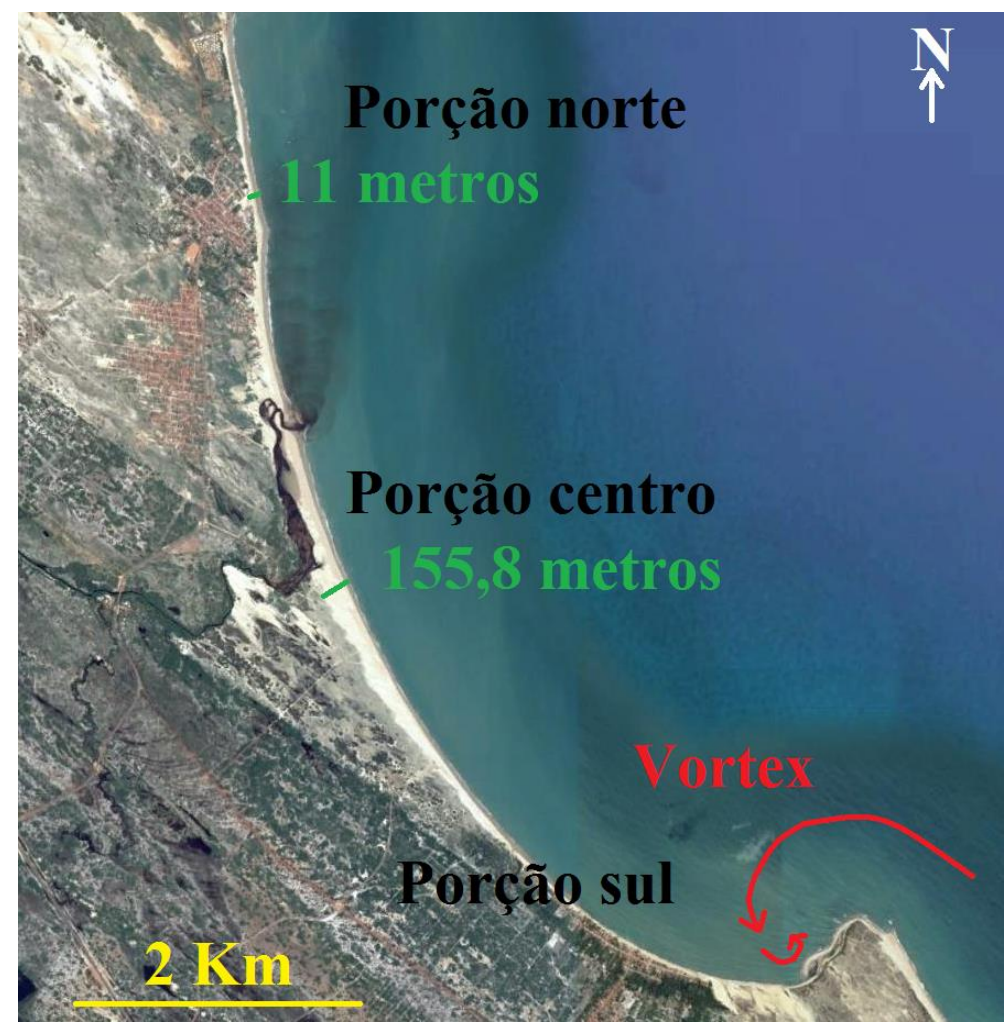

Figura 06. Imagem de satélite apresentando o vortex na cor vermelho, provocando erosão costeira na porção sul da costa do município do Rio do Fogo, enquanto que nas porções centro e norte, a linha na cor verde está demonstrando a variação da linha de costa. Fonte: Google Earth, adaptado por Camara (2016)



Figura 07. Apresentando a altura da falésia que chega a alcançar até 4 metros em relação ao nível do mar. Fonte: Camara (2016)

Essa falésia identificada pode ser um possível horst formando um Sistema de horst-gabren, onde a região do gabren foi onde se desenvolveu a planície costeira e de deflação, por ser de altitude mais baixa, próxima ao do mar. Enquanto que o horst é caracterizado pelas rochas sedimentares siliciclásticas caracterizadas como arenitos da Formação Barreiras que sofreram soerguimento devido a ativação dos sistemas de falhas neotectônicos. Esse tipo de evidência é 
bastante comum na porção sul do litoral oriental do RN, onde os sistemas de lineamentos e falhas possuem a mesma orientação que os que foram identificados no presente trabalho.

\section{CONSIDERAÇÕES FINAIS}

No presente trabalho, foi comprovado a importância de uma observação maior e um estudo mais aprofundado quando se trata da neotectônica em relação a morfologia costeira, fluvial e lacustre. Onde as unidades geoambientais são muito dinâmicas e recentes, alcançando idades entre o quarternário ao holoceno, épocas em que a ativação e reativação de falhas e tensões ocorreu, provocando forte controle no comportamento e na geomorfologia de umas unidades geoambientais, enquanto que influenciou de forma mais suave outras unidades. Por essa razão estudos mais aprofundados ainda serão realizados nessa temática para poder propiciar mais informações importantes na interação da neotectônica com esses elementos. Pois dessa forma, será possível aprimorar tanto a forma de gestão desses ambientes como também de precaver possíveis problemas ambientais ou áreas mais sensíveis por conta dessa interação da forçante endógena com o ambiente.

\section{REFERÊNCIAS}

BEZERRA, F.H.R.; Amaro, V.E.; Vita-Finzi, C; Saadi, A. 2001. Pliocene-Quaternary fault control of sedimentation and coastal plain morphology in NE Brazil. Journal of South American Earth Sciences, Volume 14, N. 1, April 2001. p. 61-75.

JAIN, E.V. The post-Paleozoic volcanism of northeast Brazil and its tectonic significance. Academia Brasileira de Ciências, 1976, 48:299-312.

TRIFONOV, V.G. Na overview of neotectonic studies. Internacional Geolic Review, 1989, 31:111-160.

OBRUCHEV, V. A. (1948). "Osnovnye cherty kinetiki i plastiki neotektonik". Izv. Akad. Nauk, Ser. Geol. 1948, p. 13-24.

\section{AGRADECIMENTOS}

Gostaria de agradecer primeiramente a Deus e aos meus pais, ao professor Cestaro, a minha colega Andreza e a toda turma da pós-graduação de geografia física da UFRN pelo apoio, carinho e dedicação para conseguirmos desenvolver essa pesquisa. Obrigado a todos.

Recebido em: 14/08/2016

Aceito para publicação em: 01/10/2016 